\title{
ARTICLE
}

Cite this: DOI: 10.1039/xoxxooooox

\section{Solution-based nanosensors for in-field detection with the naked eye}

\author{
S. Paterson ${ }^{\mathrm{a}}$ and R. de la Rica ${ }^{\mathrm{a}, *}$
}

Received ooth January 2012,

Accepted ooth January 2012

Nanomaterials are revolutionising analytical applications with low-cost tests that enable

DOI: $10.1039 / \times 0 x x 00000 x$ detecting a target molecule in a few steps and with the naked eye. With this approach, nonexperts can perform analyses on-site and without utilising electronic readers. This is advantageous in point-of-care diagnostics, in-field measurements and analyses performed in www.rsc.org/ resource-constrained settings. Here we review the main strategies adopted for detecting analytes with the naked eye and at the point of need using plasmonic nanosensors, catalytic nanoparticles and fluorescent nanomaterials. Examples of the detection of ions, glucose, small molecules, peptides and proteins with the nanosensors are explained in detail.

\section{Introduction}

The design of sensors for in-field measurements has been a central issue of analytical chemistry for decades. From the diagnosis of diseases at the point of care $^{1}$ to the detection of hazardous levels of pollutants ${ }^{2,3}$ and the identification of pathogens in food samples, ${ }^{4,5}$ there is a growing need for obtaining accurate information about the composition of a sample rapidly and at the point of need. For many years electrochemical sensors have dominated the area of in-field sensing due to the possibility of fabricating all the elements of the sensor with well-known microfabrication techniques. ${ }^{6,7}$ These fabrication methods generate portable, compact devices in which the transducers are directly integrated with the circuitry. ${ }^{8,9}$ However, although microchips containing electrochemical transducers can be mass-produced, the manufacturing cost of these devices is still too high for certain applications in which the sensor cannot be reutilised or recycled. Electrical readers are also expensive, and therefore their utilisation can only be justified in routine tests, for example in diagnostic tests required by chronic patients (e.g. diabetic patients) or in diagnostics performed in hospitals and doctor offices (Fig. 1A). ${ }^{10}$ Isolated measurements performed at the point of need (e.g. tests for sexually-transmitted diseases performed at home), or in resource-constrained settings, cannot always rely on the utilisation of such complex devices to read the signal generated by a single-use test.

A winning strategy to reduce the costs associated to the utilisation of electronic readers is to design sensors in which the signal can be detected with the naked eye. ${ }^{11}$ Usually this requires either and increase/decrease in colouration $^{12}$ or a change in the tonality of a coloured solution. ${ }^{13}$ When the difference between the colour generated by the solution containing the analyte and the colour generated by the blank solution is well defined, this method enables detecting the presence of a particular molecule without the utilisation of any external instrumentation. Frequently the results are qualitative in the form of a positive/negative outcome. This means that the test can detect the presence of the analyte when this is found at concentrations well above a threshold value, but usually it cannot provide precise, quantitative information. ${ }^{13}$ Samples detected as positive may be analysed at a later stage in centralised laboratories, where the acquisition of quantitative information is available.

Until recently, most tests for the detection of analytes with the naked eye relied in the utilisation of dipsticks and lateral flow assays. These detection schemes combine an ingenious paper-based fluidic system with a naked-eye detection mechanism in a disposable sensor. ${ }^{14}$ Reagents are stored at different positions of the paper substrate by simply spotting the chemicals and letting them dry. Liquids flow through the paper substrate after addition of a drop of sample. ${ }^{15}$ This mixes the reagents and generates the colourimetric signal (Fig. 1B). Despite the great success of this approach, it has been found that there is a great variability in the sensitivity and specificity associated to different paper-based sensing devices. ${ }^{16}$ This can easily cause false-positive and false-negative results that may have grave consequences, especially in diagnostics. One of the main sources of variability is the material of which these 


\section{ARTICLE}

A

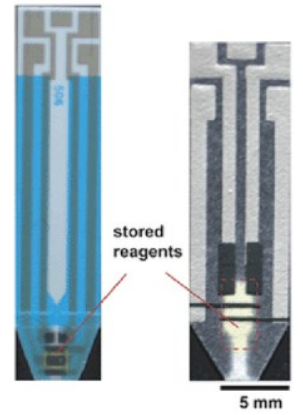

B

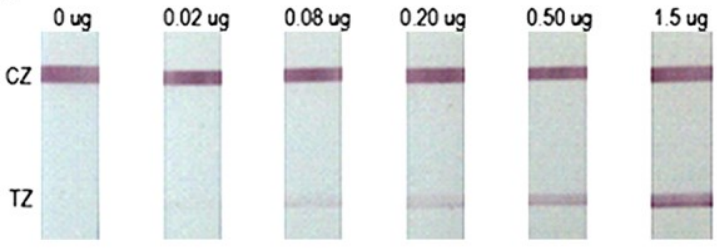

C

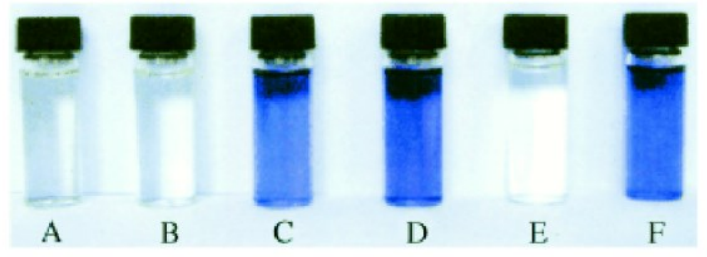

D

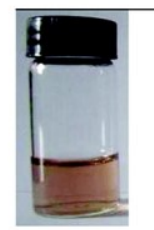

i

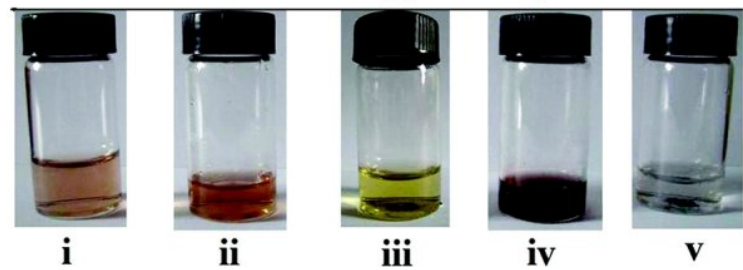

Figure 1. Current strategies for in-field detection of analytes; (A) Quantitative electrochemical detection with disposable electrodes and electronic readers (glucose meter) (reproduced from [10] with permission from the Royal Society of Chemistry); (B) Naked-eye detection with paper-based substrates: detection of histone methylation as a function of the amount of histone extract (reproduced from [20] with permission from the American Chemical Society); (C) Solution-based detection of analytes with the naked eye: specific detection of tryptophan alone (C) or in the presence of other 9 amino acids (D), that do not generate a signal by themselves (B) (reproduced from [21] with permission from Willey-VCH); (D) Solution-based detection of analytes with nanoparticles: detection of aromatic isomers with cyclodextrin-modified silver nanoparticles; top: phenol (ii), pyrocatechin (iii), hydroquinone (iv), resorcinol (v); bottom: aniline (ii), o-phenylenediamine (iii), p-phenylenediamine (iv), and mphenylenediamine (v) (reproduced from [31] with permission from the American Chemical Society).

devices are fabricated: different paper substrates have different flow speeds, which are crucial to determine the sensitivity and specificity of the analysis. ${ }^{17}$ Dipsticks and lateral flow assays have the added issue of not being very sensitive. ${ }^{18}$ Although some methods have been proposed to improve the sensitivity of these tests several orders of magnitude, they rely on multi-step procedures that are difficult to implement in in-field measurements. ${ }^{19,20}$

A potential solution to reduce variability issues related to the utilisation of solid substrates is to perform the test in solution. In this approach, a drop of sample is added to a solution containing the sensor and the presence of the analyte generates a signal that can be detected with the naked eye. For example a solution containing formic acid and $\mathrm{HCl}$ generates a violet-blue colour in the presence of the amino acid tryptophan (Fig. 1C). ${ }^{21}$ The reaction is highly specific and allows detecting the target amino acid even in the presence of a mixture of other amino acids. This type of solution-based sensor is single-use, disposable and does not require electric readers to detect the presence of the target molecule. Yet, this approach seems to be restricted to straightforward detection mechanisms in which the target molecule reacts in a selective manner with a component of the solution to generate a colourimetric signal. This is a limitation compared to paper-based sensors, which enable complex detection schemes such as immunoassays ${ }^{22}$ and DNA tests. $^{21}$

In recent years nanosensors have been changing this paradigm by enabling the detection of a multitude of analytes in solution and with the naked eye (Fig. 1D). ${ }^{23-25} \mathrm{~A}$ key factor for the development of complex sensing approaches is that these nanomaterials can be decorated with myriads of biomolecules, ${ }^{26-28}$ polymers $^{29}$ and macrocycles ${ }^{30,31}$ that confer them specific recognition capabilities for the detection of a wide array of molecules. For example, nanoparticle aggregates linked by peptides can be used for the one-step detection of proteases when the protease activity triggers the dispersion of the nanoparticle collectives. ${ }^{32,33}$ Nanoparticles modified with macrocycles such as cyclodextrins and cucurbiturils can be used to detect enzymatic activity. ${ }^{34,35}$ Solutions containing nanomaterials modified with oligonucleotides can be used to detect genetic mutations, ${ }^{36-38}$ malfunctioning enzymes ${ }^{39}$ and even hazardous heavy metals. ${ }^{25,40}$ Moreover, nanoparticles possess extraordinary optical properties derived from their nanoscale dimensions that facilitate the observation of small changes in the concentration of analytes with the naked eye. With plasmonic nanosensors, the colour of the nanoparticle solution can be tuned by changing the composition and morphology of the nanomaterials. ${ }^{41-43}$ Semiconducting 
nanoparticles show size-dependent emission properties that cover the whole visible spectrum. ${ }^{44,45}$ Nanomaterials have been a true game changer in the field of solution-based sensing due to their easy functionalization, chemical reactivity and outstanding physical properties.

In this manuscript we will review different solution-based strategies for the naked-eye detection of analytes with nanomaterials. After giving an overview of the different signal generation mechanisms available for naked-eye detection, we will show some key examples of solution-based nanosensors classified as a function of the type of analyte detected. In these examples we will focus on approaches that allow the detection of the target molecule at the point of need without the utilisation of laboratory-based methods. For example, methods for the naked-eye detection of nucleic acids will not be considered because the detection of nucleic acids usually involves their extraction from cells with multi-step protocols that should be performed in a well-equipped laboratory. Dipsticks, lateral flow and paper-based assays are out of the scope of this manuscript. Several excellent recent reviews covering these approaches are available in the literature. ${ }^{18,19,22,46}$

\section{Signal generation mechanisms}

In this section we will review different approaches for the generation of signals detectable with the naked eye using nanosensors. We will first overview the physicochemical properties of a particular family of nanomaterials. Then we will explain several strategies commonly utilised to implement these physicochemical properties in the design of sensors for the naked-eye detection of analytes. Plasmonic, catalytic and fluorescent nanoparticles will be the focus of our study.

\section{Plasmonic nanosensors}

The localised surface plasmon resonance (LSPR) of noble metal nanoparticles is the origin of the intense colourations usually observed in nanoparticle solutions. ${ }^{47}$ The LSPR is affected by the size, shape and state of aggregation of the nanoparticles as well as by the dielectric properties of the surrounding medium. ${ }^{48,49}$ This observation has been utilised to design myriads of colourimetric sensors in which the presence of the analyte changes the LSPR of plasmonic nanosensors and generates a change in the colour of the nanoparticle solution that can be detected with the naked eye.

For example, it is well established that the LSPR of noble metal nanoparticles shifts to higher wavelengths when the nanoparticles assemble into aggregates. The aggregation of spherical gold nanoparticles usually results in a transition from red to purple or deep blue colour, ${ }^{50}$ whereas the aggregation of silver nanoparticles usually changes the colour of the solution from yellow to brown or grey (Figs. 2A and 2B). ${ }^{51}$ If the aggregates are large enough the nanoparticle networks can sediment, which results in an apparent decrease of the colouration of the solution as the nanoparticles accumulate at the bottom of the test tube. ${ }^{52,53}$ Thus, the signal generated by the assembly of plasmonic nanosensors can be either a change in the tonality of the solution or a change in its colour intensity. It should be noted that the most widely available citratestabilised nanoparticles usually aggregate in solutions containing highly concentrated ions. This can be prevented by covering the surface of the nanoparticles with hydrophilic ligands. ${ }^{54}$ For example, commercially available polyethylene glycol molecules can be used to prevent non-specific aggregation issues. These molecules are usually anchored to the nanoparticles by thiol moieties that have high affinity for metal surfaces. ${ }^{55}$

Triggering the aggregation or dispersion of nanoparticles is not the only strategy available to generate colourimetric signals in biosensing. In the last years, a new trend has been studying the growth of nanoparticles with different morphology and composition as a way to tune the LSPR of the nanosensors. ${ }^{42,56,57}$ For example tuning the kinetics of growth of gold nanoparticles with an enzyme can result in the growth of de novo aggregated or non-aggregated nanoparticles with easily identifiable blue or red tonalities. ${ }^{58}$ The LSPR of gold nanostars can be tuned by reducing silver on their surface with an enzyme-guided crystal growth process. ${ }^{42}$ The colourimetric signal of plasmonic nanosensors can be greatly amplified by reducing gold ions on gold seeds with hydroxylamine, ${ }^{59}$ or with hydrogen peroxide generated by the enzyme glucose oxidase (Fig. 2C). ${ }^{57}$ Despite the great interest in these approaches, which may result in extremely low limits of detection and ultrahigh sensitivities, the utilisation of solutions containing metal ion precursors for growing the nanoparticles makes these approaches unsuitable for many in-field detection approaches, since these solutions are generally not stable in ambient conditions for extended periods of time. ${ }^{13}$
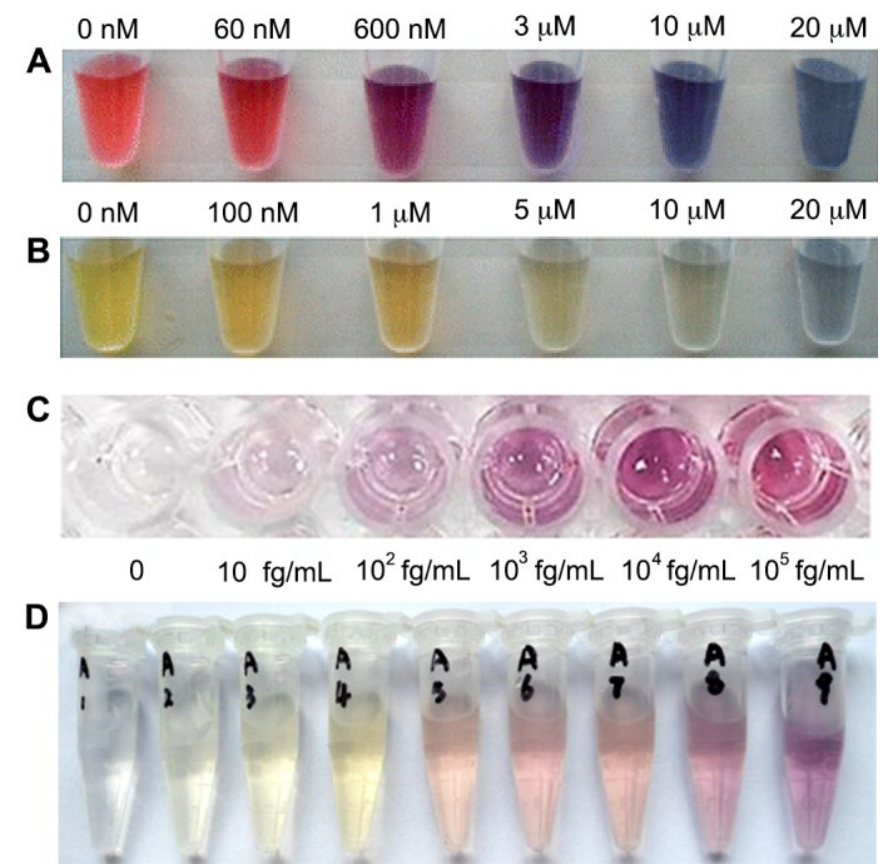

Figure 2. Tuning the LSPR of noble metal nanoparticles for the detection of analytes with the naked eye; (A) Aggregation of DNA-modified gold nanoparticles in the presence of different concentration of $\mathrm{Ag}^{+}$changes the colour of the solution from red- to blue-coloured; (B) Aggregation of DNA-modified silver nanoparticles in the presence of different concentration of $\mathrm{Hg}^{2+}$ changes the colour of the solution from yellow- to greycoloured (reproduced from [51] with permission from the Royal Society of Chemistry); (C) Enlargement of gold seeds by glucose oxidase for the detection of prostate specific antigen (PSA) at different concentrations (reproduced from [57] with permission from the ACS); (D) Etching of silver nanoplates in the presence of $5 \cdot 10^{-7} \mathrm{M}$ iodide with different concentration of nanoplates (increasing concentration from A1 to A9) (reproduced from [84] with permission from Elsevier). 
Another interesting phenomenon in nanochemistry that has been utilised as the signal generation step in sensing is nanoparticle etching. ${ }^{60-62}$ This usually involves the oxidation of silver nanoparticles to silver ions. The etching of spherical silver nanoparticles results in a decrease of the colouration of the nanoparticle solution or in the aggregation of the nanoparticles (change in tonality). ${ }^{63}$ Gold nanoshells containing a silver alloy have also been used as nanosensors. ${ }^{64}$ The oxidation of the silver in the alloy results in a change of the composition of the shell that generates a change in the tonality of the nanoparticle solution. Nanoparticles with sharp edges and spiky shapes are prone to reshape into spherical nanoparticles. ${ }^{65}$ Etching of concave edges results in a change in the morphology-related LSPR of the nanoparticles that sometimes can be detected with the naked eye. Triangular silver nanoplates, also called silver nanoprisms, can reshape in the presence of hydrogen peroxide or halides to yield silver nanodisks. ${ }^{23,62,66}$ The change in shape from the sharp edges of the triangles to the rounded corners of spherical nanoparticles changes the colour of the solution from blue/purple to red or yellow-coloured depending on the concentration of the analyte (Fig. 2D).

\section{Catalytic nanomaterials}

One of the most commonly utilised methods for generating colourimetric signals in biosensing consists in generating coloured molecules with the enzyme horseradish peroxidase (HRP). ${ }^{67}$ This type of signal generation mechanism has the advantage of amplifying the signal through the biocatalytic property of the enzyme. Unfortunately HRP can easily become inactive due to denaturation at high temperatures, digestion by proteases or degradation by microorganisms, among other factors. These factors can have a great impact in in-field measurements, when the sensors cannot be kept in ideal conditions.

Some noble metal nanoparticles have been found to possess catalytic oxidation properties that mimic those of HRP. Since inorganic nanomaterials usually have a longer shelf life compared to biomolecules, the utilization of catalytic nanoparticles can overcome the limitation of using HRP as the signal generation step in in-field detection schemes. Platinum, ${ }^{68}$ palladium, ${ }^{69}$ gold, ${ }^{70}$ carbon, ${ }^{71} \mathrm{CeO}_{2}{ }^{72}$ and $\mathrm{Fe}_{3} \mathrm{O}_{4}{ }^{73}$ nanoparticles have been shown to oxidase peroxidase substrates such as 3,3,5,5-tetramethylbenzidine (TMB) in the presence of hydrogen peroxide. The oxidation of TMB generates bluecoloured solutions. The reaction can be stopped by adding concentrated $\mathrm{H}_{2} \mathrm{SO}_{4}$, which also triggers a change in the colour of the solution from blue- to yellow-coloured (Fig. 3A).

Gold nanoparticles have also been found to mimic the activity of the enzyme glucose oxidase (GOx) by catalysing the conversion of glucose into gluconic acid and hydrogen peroxide in the presence of oxygen (Fig. 3B). ${ }^{74}$ In this study it was proposed that the ligands around the gold nanoparticles played a crucial role in defining the catalytic properties of these nanomaterials. The hydrogen peroxide generated by the nanoparticles can be used by HRP to generate coloured signals as explained above. GOx and GOx mimics can be used for the detection of glucose in diabetic patients ${ }^{23}$ or in enzyme-linked immunoassays for the detection of proteins with antibodies as biorecognition elements. ${ }^{42,57}$
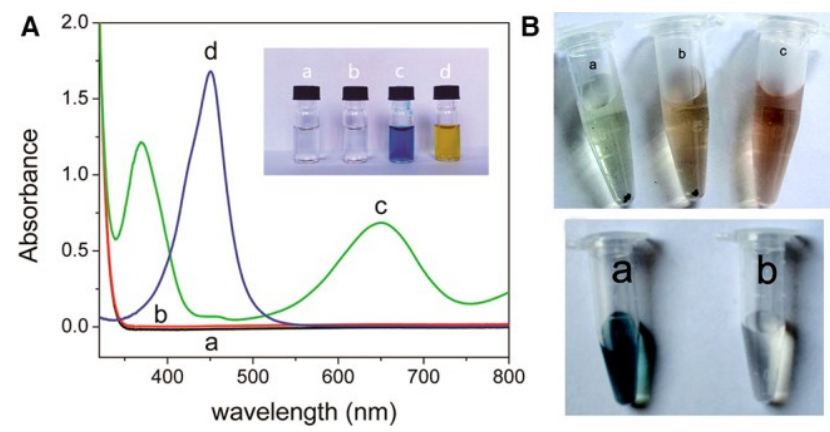

Figure 3. Generation of colourimetric signals with catalytic nanoparticles; (A) The oxidation of TMB by porous platinum nanoparticles grown on graphene oxide changes the colour of the solution to blue-coloured (c), which turns yellow-coloured upon addition of $\mathrm{H}_{2} \mathrm{SO}_{4}$ (d) (reproduced from [68] with permission from the American Chemical Society); (B) Top: Addition of glucose to gold nanoparticles (b) or glucose oxidase (c) generates a red-coloured solution due to reaction of the as-generated gluconic acid with hydroxamine and Fe(III); Bottom: (a) the GOx-like activity of gold nanoparticles generates hydrogen peroxide that is used by HRP to oxidise ABTS $^{2-}$ (green coloured solution) (reproduced from [74] with permission from the American Chemical Society).

\section{Fluorescent nanosensors}

Fluorescent nanosensors for naked-eye detection require, at least, the utilisation of a UV lamp in order to excite the fluorophores and detect the fluorescent signal. This condition makes this approach less straightforward than detection systems based in the detection of colours, which do not require any external instrument to read the signal. Nevertheless, UV lamps are small, cheap and easy to manipulate and their utilisation may be justified in situations where bulky or expensive instruments are not available. Another difficulty faced by fluorescent nanosensors is that the emission of many fluorophores can be quenched or bleached by several external factors (presence of oxygen, direct contact with light), and therefore it should always be compared with a standard.

The utilisation of nanosensors can partially alleviate the stability problems found in fluorescent sensors for in-field measurements. Quantum dots are luminescent semiconducting nanocrystals whose emission wavelength is intimately to their size, that is, they can be fabricated with different sizes emitting in different spectral regions. ${ }^{44}$ Quantum dots show broad absorption spectra, narrow and size-tuneable emission peaks and large Stokes shifts. These features make quantum dots perfect donors in fluorescence resonance energy transfer (FRET). ${ }^{75}$ The broad adsorption spectrum of quantum dots makes it easy to visualise their emission with a conventional UV lamp. Furthermore, quantum dots are less sensitive to photobleaching compared to many organic fluorophores.

Utilising energy transfer phenomena can also be helpful to provide an inner standard for fluorescence measurements. In this context, "switch-on" approaches based in energy transfer between quantum dots and molecular fluorophores have been proposed. ${ }^{76}$ In these nanosensors, the fluorescence of quantum dots is quenched by a ruthenium complex attached to the surface of the nanocrystals. The analyte triggers the removal of the complex from the surface. This disrupts the energy transfer between the quantum dots and the ruthenium complex and the fluorescence of the quantum dots can be observed ("switches 
on"). Other nanosensors designs consist in triggering the aggregation of the quantum dots in the presence of the analyte. ${ }^{33}$ This results in an exciton energy transfer process that switches off the photoluminescence of the nanocrystals.

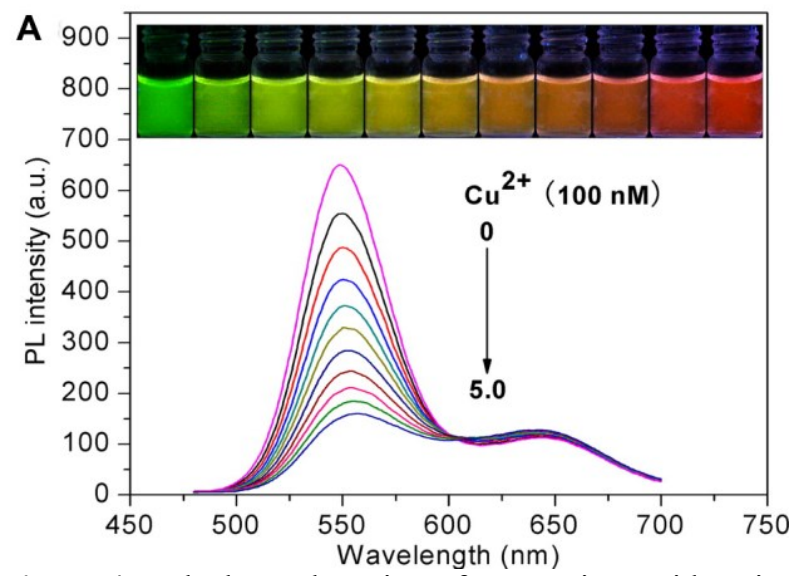

Figure 4. Naked-eye detection of copper ions with ratiometric fluorescent nanosensors. Green-emitting quantum dots are quenched by increasing concentrations of copper ions. This reveals the red emission of fluorescent nanoparticles buried in the core of the nanosensors (reproduced from [77] with permission from the American Chemical Society).

One of the most elegant approaches for the naked-eye detection of analytes with quantum dots consists in fabricating fluorescent ratiometric probes. ${ }^{77}$ In this approach, red-emitting quantum dots are confined to the core of silicon dioxide nanoparticles. Green-emitting quantum dots are then attached to the surface of the silicon dioxide nanoparticles. The photoluminescence of the quantum dots is quenched by the presence of copper ions, which are the target analyte. However, red-emitting quantum dots are not affected by copper ions because they are protected by the transparent silicon dioxide nanoparticle. Therefore the presence of copper ions quenches only the green emission and reveals the red emission of quantum dots at the core of the nanoparticles, which act as an internal reference (Fig. 4).

\section{In-field detection of analytes with the naked eye.}

In the following sections we review some key examples of the application of nanomaterials for the detection of analytes at the point of need. These examples are classified by the type of analyte detected.

\section{Ions}

Real-time, on-site detection system for ions such as the heavy metals, $\mathrm{Mg}^{2+}, \mathrm{Hg}^{2+}, \mathrm{Pb}^{2+}, \mathrm{Al}^{3+}, \mathrm{Cu}^{2+}, \mathrm{Cr}^{3}$ are required in environmental monitoring and toxicology. Sensors for the detection of heavy metals must be highly selective, sensitive and fast to compete with laboratory-based methods.

Mercury has garnered widespread attention for colourimetric detection systems as it has been found to be one of the most dangerous metal elements to human health and environmental safety. The most common form of mercury is methyl mercury, which can bioaccumulate through the food chain from aquatic systems. ${ }^{78}$ Mirkin et al have pioneered the colourimetric detection of mercury with plasmonic nanoparticles. ${ }^{79}$ They utilized DNA-functionalized gold nanoparticles as nanosensors. It is known that mercury ions can bridge a $\mathrm{T}-\mathrm{T}$ mismatch by means of the thymine- $\mathrm{Hg}^{2+}$-thymine coordination chemistry, which triggers the aggregation of the DNA-covered nanoparticles in the presence of $\mathrm{Hg}^{2+}{ }^{80}$ It was hypothesized that $\mathrm{Hg}^{2+}$ binding to the $\mathrm{T}-\mathrm{T}$ mismatch sites would cause an increase in the melting temperature, $\mathrm{T}_{\mathrm{m}}$, of the DNA. This was observed by increasing the temperature of the solution and observing the melting point of the nanoparticle aggregates as the colour change was reversed to red-mauve. It was found that there was a direct relationship between the concentration of $\mathrm{Hg}^{2+}$ and $\mathrm{T}_{\mathrm{m}}$. With this method, $\mathrm{Hg}^{2+}$ could be quantified with high specificity and sensitivity with lower detection limits than previously reported approaches. The selectivity of the system was further tested against environmentally relevant ions and it was found that only solutions containing $\mathrm{Hg}^{2+}$ had a significantly higher $\mathrm{T}_{\mathrm{m}}$ than samples containing other ions. At $47^{\circ} \mathrm{C}$ the samples containing $\mathrm{Hg}^{2+}$ remained purple whereas, the solutions containing other ions were red (Fig. 5A). This has been found to be a promising progress for the detection of $\mathrm{Hg}^{2+}$. However, it should be noted that measuring $\mathrm{T}_{\mathrm{m}}$ is not suitable for in-field measurements, since additional equipment is needed to control the temperature.

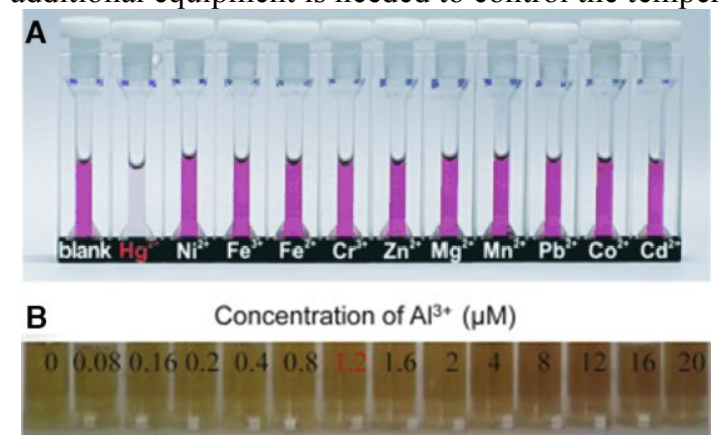

Figure 5. Detection of ions with nanosensors; (A) detection of $\mathrm{Hg}^{2+}$ with DNA-decorated gold nanoparticles at $45^{\circ} \mathrm{C}$ showing the high specificity for the target analyte (reproduced from [79] with permission from Willey-VCH); (B) detection of $\mathrm{Al}^{3+}$ at different concentrations with silver nanoparticles modified with glutathione (reproduced from [81] with permission from Elsevier).

$\mathrm{Wu}$ et al utilised glutathione-functionalized silver nanoparticles (GSH-AgNPs) to quantify the levels of $\mathrm{Al}^{3+}$ in the presence of 1-cysteine (Cys). ${ }^{81} \mathrm{GSH}$ absorbs onto the surface of the $\mathrm{Ag}$ nanoparticle via its thiol group. As $\mathrm{Al}^{3+}$ forms complexes with GSH, aggregation occurs, which results in a colour change from yellow to reddish brown or red observable by naked eye (Fig. 5B). Control experiments of $\mathrm{Al}^{3+}$ incubated with GSH-AgNPs but without Cys did not yield any observable colour change even at high concentrations of $\mathrm{Al}^{3+}$. The performance of the nanosensors was tested in real water (tap water or lake water). With these samples it was found that the limit of detection with the naked eye was $1.2 \mu \mathrm{M}$, which is far lower than the set national drinking water standard. The selectivity of this system may be questioned since, although it tested well against other ions at concentrations of $20 \mu \mathrm{M}$, there have been previously reported studies of glutathionefunctionalized gold nanoparticles for the detection of lead ions with a similar method that led to a positive response. ${ }^{82}$ However, the previous study did not test for the detection of $\mathrm{Al}^{3+}$.

Another naked-eye detection method for the detection of ions that does not rely on particle aggregation consists in changing the morphology of silver nanoparticles. The strong 
LSPR of silver triangular nanoplates (Ag-TNPs) can be adjusted throughout the visible and near-infrared regions by manipulating the edge, tip edge and length of these nanomaterials. ${ }^{83}$ Cao and Ling reported that Ag-TNPs can be reshaped into nanoparticles in the presence of iodide. ${ }^{84}$ The monitoring of iodide is essential in food and drugs as it plays a crucial role in thyroid functionality and neurological activity. Solutions containing Ag-TNPs were mixed with different concentrations of iodide and within 30 minutes a distinctive colour change could be seen from blue- to yellow-coloured. TEM confirmed the morphology of the Ag-TNPs changed to nanoparticles. As the concentration of iodide was directly proportional to the concentration of silver nanoparticles in solution, the aim was to find the critical concentration of iodide to Ag-TNPs in which an obvious colour change could be observed. The lowest detectable concentration of iodide measured by UV-vis absorption was $8.8 \mathrm{nM}$, the lowest detectable concentration by naked eye was found to be approximately $0.1 \mu \mathrm{M}$. Selectivity tests conducted against several other anions showed that bromide had similar results to iodide although at 10-fold higher concentrations. However, posterior optimization of the same detection system via the addition of $\mathrm{Na}_{2} \mathrm{~S}_{2} \mathrm{O}_{3}$ led to better selectivity towards iodide with the same sensitivity. ${ }^{85}$

\section{Glucose}

The detection of glucose is crucial in a wide range of fields such as clinical diagnostics, ${ }^{86}$ food industries ${ }^{87}$ and biotechnology. ${ }^{88}$ Perhaps the most recognized application of glucose sensors is for the diagnosis and management of diabetes mellitus, which is characterised by varying levels of glucose. ${ }^{89}$ According to the World Health Organization (WHO), diabetes affects 347 million people worldwide and has been estimated that it will be the 7 th leading cause of death by 2030. Currently, the most common detection method for glucose is an electrochemical biosensor based in the biocatalytic conversion of glucose by the enzyme glucose oxidase $(\mathrm{GOx}) .{ }^{90}$ This biosensor requires an electronic reader to transduce the signal. In developing countries these electronic readers may be too costly for routine testing. In this context a naked-eye detection system that does not require an electronic readout could provide a much simpler and economical method for glucose sensing.

GOx can be used for the naked-eye detection of glucose when the production of $\mathrm{H}_{2} \mathrm{O}_{2}$ by the enzyme is used to etch nanoparticles. This causes a distinct change in morphology that translates into a change in colour. ${ }^{91}$ One advancement has been made by Xia et al, who reported a visible colour change in solutions containing silver triangular nanoplates in the presence of glucose and GOx. ${ }^{23}$ Firstly, a homogenous solution containing nanoplates and GOx was prepared. The addition of glucose resulted in the consequential enzymatic oxidation of this molecule to generate hydrogen peroxide and gluconic acid. The peroxide produced this way etched onto the blue triangle nanoplates. This resulted in a change in morphology from triangular nanoplate to nanodisc that in turn resulted in a change in colour from blue to mauve. This was found to be highly sensitive with only $10-20 \mu \mathrm{L}$ of serum required for testing. The detection limit was $0.2 \mu \mathrm{M}$, which was found to be lower than previous studies involving metal-GOx methods.

Another simple and cost effective method for detecting glucose employed bimetallic $\mathrm{Ag} / \mathrm{Au}$ nanoshells as nanosensors. ${ }^{64}$ in this approach, GOx was immobilized onto the $\mathrm{Ag} / \mathrm{Au}$ nanoshell surface followed by the addition of glucose.
This resulted in the etching of the silver, which triggered a change in the colour of the solution from red- to blue-coloured. This was caused by the removal of $\mathrm{Ag}$ by $\mathrm{H}_{2} \mathrm{O}_{2}$ from the bimetallic shell. However, this method was found to be less sensitive at high concentrations of glucose. This was due to unwarranted by-products of $\mathrm{AgOH}$ and $\mathrm{AgO}_{2}$ and possible oxidation of Ag on the nanosphere shell.

Catalytic nanoparticles can also be used for the colourimetric detection of glucose. For example it has been demonstrated that 5,10,15,20-tetrakis(4-carboxyphenyl)porphyrin-functionalized $\mathrm{Fe}_{3} \mathrm{O}_{4}$ nanocomposites $\left(\mathrm{H}_{2} \mathrm{TCPP}-\right.$ $\mathrm{Fe}_{3} \mathrm{O}_{4}$ ) show ultra-high peroxidase activity. ${ }^{92}$ This peroxidemimicking property can be used to oxidise TMB molecules in the presence of hydrogen peroxide, therefore generating a green-coloured solution that can be detected with the naked eye. When the catalytic activity of the nanoparticles was coupled with the generation of hydrogen peroxide by glucose oxidase the proposed method could detect glucose at the concentration of $0.005 \mathrm{mM}$ or higher with the naked eye.

\section{Small molecules}

Mirkin and coworkers utilised oligonucleotide-covered nanoparticles for the selective detection of the amino acid cysteine. ${ }^{93}$ The detection of cysteine is required in diagnostics and usually requires complicated instrumentation. The nanosensors were assembled via hybridisation and highly stabilising thymine- $\mathrm{Hg}^{2+}$-thymine coordination bonds, as explained in the "Ions" section (Fig. 5A). Cysteine has high affinity for $\mathrm{Hg}^{2+}$. Therefore the analyte competes with thymine for the interaction with $\mathrm{Hg}^{2+}$. This leads to the disassembly of the nanoparticles and the observation of a red-pink coloured solution. The proposed method allowed detecting cysteine at the ultralow concentration of $100 \mathrm{nM}$ and was highly selective towards different types of amino acids.

Oligonucleotide aptamers that specifically recognise bisphenol A (BPA) have been used for the in-field detection of this toxic molecule. ${ }^{94}$ In this approach gold nanoparticles were modified with the following aptamer sequence: 5CCGGTGGGTGGTCAGGTGGGATAGCGTTCCGCGTATG GCCCAGCGCATCACGGGTTCGCACCA-3. In the absence of BPA the aptamer acts as stabilising agent that prevents saltinduced aggregation due to the negatively charge phosphate groups in the oligonucleotides. However it was found that the interaction with BPA decreased the salt tolerance, which caused the nanoparticles to aggregate. This enabled the detection of BPA with the naked eye at concentrations higher than $5 \mathrm{ng} / \mathrm{mL}$ in tap water and with a one-step method (Fig. 6A).

Melamine is a chemical that is illegally added to milk products and that can result in renal failure or death in infants. ${ }^{95}$ Consequently, a fast method for the detection of melamine is required for detecting the fraudulent use of this hazardous molecule in the food industry. This can be achieved with gold nanoparticles modified with the molecule 1-(2-mercaptoethyl)1,3,5-triazinane-2,4,6-trione (MTT). ${ }^{96}$ Hydrogen bonding between this molecule and melamine triggers the aggregation of the nanoparticles. With this method it is possible to detect melamine with a limit of detection of $20 \mathrm{nM}(2.5 \mathrm{ppb})$, which is 3 orders of magnitude lower than the safety limit allowed by the U.S. Food and Drug Administration (Fig. 6B). It was also found that milk did not change the performance of the nanosensors, which makes this approach promising for the detection of melamine in infant formula. 

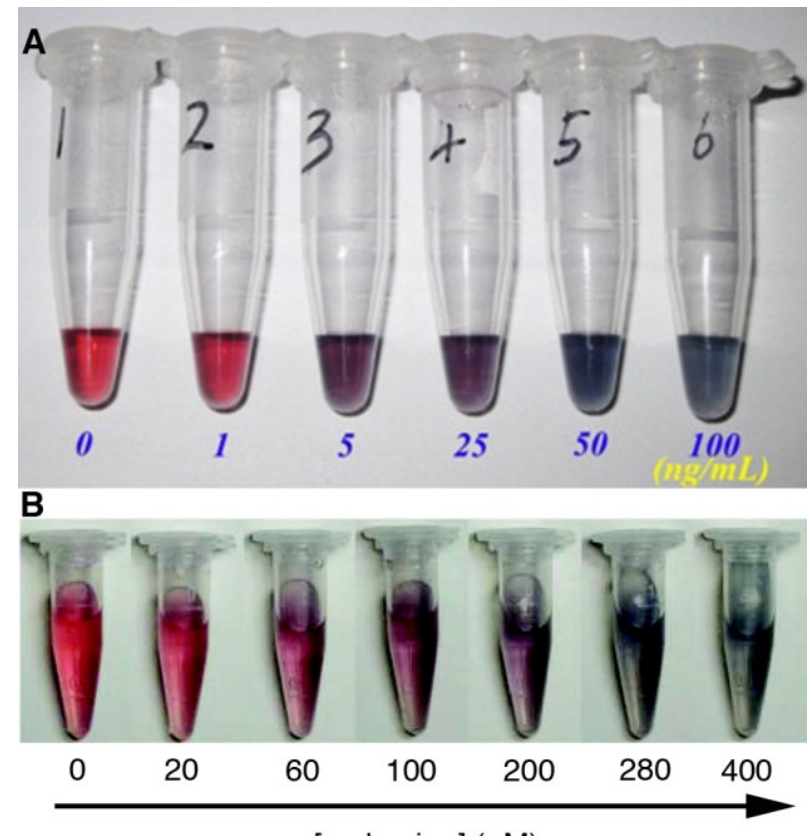

[melamine] ( $\mathrm{nM})$

Figure 6. Naked-eye detection of bisphenol A (BPA) in tap water (A, reproduced from [94] with permission from Elsevier) and melamine (B, reproduced from [96] with permission from the American Chemical Society) with gold nanoparticles.

\section{Peptides and proteins}

Classical methods for the detection of proteins usually require utilising antibodies as biorecognition elements. Since many nanoparticle-based biosensors are based in the aggregation of the nanosensors, it should be straightforward to design colourimetric detection systems based on the aggregation of antibody-modified nanoparticles in the presence of a target proteins. Yet, proteins (including antibodies) are rather bulky, and therefore nanoparticles in assemblies connected by antibody-antigen interactions may not be close enough to trigger large spectral shifts. This may limit the sensitivity of the biosensor. Nevertheless, some elegant examples of biosensors using this procedure exist in the literature. For example it was proposed that antibody-covered gold nanoparticles can aggregate in the presence of the virus bacteriophage $\mathrm{T} 7$, which causes a change in the colour of the solution from red- to blue-coloured. ${ }^{97}$ In another example antibody-covered gold nanoparticles were able to aggregate peptide-covered nanoparticles. ${ }^{53}$ Rather than changing the colour from red to blue, the aggregation process made the nanoparticles sediment, therefore resulting in a decreased colouration of the nanoparticle solution.

A successful strategy for detecting proteins with nanosensors consists in controlling the state of aggregation of the nanoparticles with a positively charged biomolecule or polymer. Positively charged molecules induce the aggregation of the nanoparticles. In this detection scheme, the biomolecule or polymer can establish physical interactions with the target molecule, for example electrostatic intereactions. This prevents the aggregation of the nanoparticles by the positively charged biomolecule or polymer. For example, the cancer-related protein cyclin $\mathrm{A}_{2}$ interacts with the cationic peptide RWIMYF$\mathrm{NH}_{2} \cdot{ }^{98}$ In the absence of cyclin $\mathrm{A}_{2}$ the cationic peptide promotes the aggregation of the nanoparticles and the solution turns blue (gold nanoparticles) or brown (silver nanoparticles).
In the presence of the target protein the nanoparticles remain separated and the solution is red-coloured (gold) or yellowcoloured (silver). This strategy enabled detecting cyclin $\mathrm{A}_{2}$ with the concentration of $40 \mathrm{nM}$ with the naked eye and with a simplified procedure compared to commonly used ELISA for the detection of this analyte.

A similar approach has been adopted for detecting thrombin, a protein implicated in blood coagulation, thrombosis, inflammation, angiogenesis, tumour growth and metastasis. $^{99}$ In this approach, the aggregation of gold nanoparticles is triggered by the positively-charged polymer PDDA (poly(diallyldimethylammoniumchloride)). The thrombin binding aptamer (TBA) is a negatively charged oligonucleotide with sequence 5'TTTGGTTGGTGTGGTTGGTTT-3' that has high affinity for thrombin. In the absence of thrombin, PDDA interacts with TBA, which prevents the aggregation of the nanoparticles. In the presence of thrombin, the aptamer interacts with it and the PDDA aggregates the nanoparticles. With this method it is possible to detect thrombin with the concentration of $10 \mathrm{pM}$ even when spiked into a complex matrix such as serum.

\section{A}
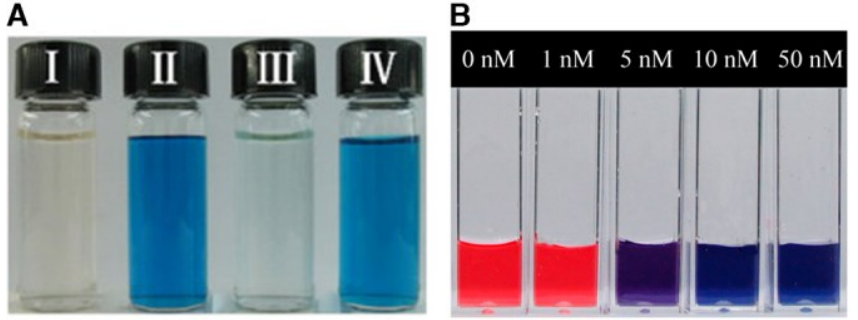

Figure 7. Strategies for the naked-eye detection of proteins; (A) detection of prion proteins with quantum dots; (I) orange quantum dots; (II) green-blue malachite green; (III) mixture of both components; (IV) the solution turns blue in the presence of prion proteins; (reproduced from [100] with permission from Elsevier); (B) Detection of cathepsin B at different concentrations via aggregation of gold nanoparticles (reproduced from [101] with permission from the American Chemical Society).

Another interesting strategy for the detection of the proteins with nanosensors consists in using the colour complementarity principle (Fig. 7A). ${ }^{100}$ In this approach orange quantum dots are mixed with green-blue malachite green. Due to the colour complementarity principle the resulting solution appears transparent (Fig. 7A, III). Addition of a prion protein causes the aggregation of the quantum dots, which results in a decrease of the quantum dot concentration. In turn, this increases blue light that cuts through the solution, and the solution becomes bluecoloured (Fig. 7A, IV).

Finally, it should be noted that is possible to detect enzymes with the naked eye when the enzymatic activity controls the assembly or disassembly of plasmonic nanosensors. For example it has been shown that the protease cathepsin B can be detected with high sensitivity and specificity when the enzyme hydrolyses the substrate N,N'-diBoc-dityrosine-glycinephenylalanine-3-(methylthio)-propylamine (DBDY-Gly-PheMTPA) in the presence of gold nanoparticles. ${ }^{101}$ The resulting product of the enzymatic reaction (Phe-MTPA) is positively charged and can induce the aggregation of the gold nanoparticles, which results in the characteristic change in the colour of the solution from red- to blue-coloured (Fig. 7B). The assay could be used not only to quantify the levels of cathepsin $\mathrm{B}$ but also to measure the inhibitory power of different small molecules, which could be used as anti-cancer agents. 


\section{Conclusions}

Nanosensors are challenging traditional detection systems with simple tests that only require a few steps for the detection of a target molecule. This is in stark contrast with laboratorybased approaches that require hour-long incubation steps and that are not suitable for in-field measurements. Furthermore, the outstanding optical and catalytic properties of noble metal and semiconducting nanoparticles enable detecting the analyte with the naked eye. This elicits the utilisation of electronic readers, which is also a great advantage for detecting analytes at the point of need. Ions, glucose, small molecules, peptides and proteins can potentially be detected on-site with this strategy.

Despite the great promise of nanosensors for in-field measurements several aspects related to this application need to be addressed before this sensing platform can be used at the point of need. For example bare nanoparticles tend to aggregate when left in solution for prolonged periods of time due to their high surface energy. The non-specific aggregation of nanoparticles could have a great impact in detection mechanisms based in the assembly or disassembly of nanosensors. Biomolecular ligands such as the DNA molecules used for $\mathrm{Hg}^{2+}$ sensing can be hydrolised by contaminating nucleases or microorganisms. ${ }^{79}$ The performance of catalytic nanoparticles may be affected by the physisorption of contaminants on their surface. ${ }^{74}$ Long-term studies on the performance of nanosensors are required in order to determine the impact of these factors in the shelf life of the tests.

\section{Acknowledgements}

We acknowledge financial support from Tenovus Scotland.

\section{Notes and references}

${ }^{a}$ Centre for Molecular Nanometrology, WestCHEM, Pure and Applied Chemistry, University of Strathclyde, 295 Cathedral Street, Glasgow G1 1XL, U.K.

$\dagger$ Footnotes should appear here. These might include comments relevant to but not central to the matter under discussion, limited experimental and spectral data, and crystallographic data.

1. S. Boppart and R. Richards-Kortum, Sci. Transl. Med., 2014, 6 , 253rv2-253rv2.

2. R. de la Rica, E. Mendoza, and H. Matsui, Small, 2010, 6, 1753-6.

3. E. Cho, J. Kim, B. Tejerina, and T. Hermans, Nat. Mater., 2012, 11, 978-985.

4. R. de la Rica, A. Baldi, C. Fernández-Sánchez, and H. Matsui, Anal. Chem., 2009, 81, 7732-7736.

5. B. Lam, J. Das, R. Holmes, and L. Live, Nat. Commun., 2013, 4, 2001.

6. R. De La Rica, A. Baldi, C. Fernández-Sánchez, and H. Matsui, Anal. Chem., 2009, 81, 3830-3835.

$7 . \quad$ H. C. Koydemir, H. Kulah, A. Alp, A. H. Uner, G. Hascelik, and C. Ozgen, IEEE Sens. J., 2014, 14, 1844-1851.

8. S. Carrara, S. Ghoreishizadeh, J. Olivo, I. Taurino, C. Baj-Rossi, A. Cavallini, M. O. de Beeck, C. Dehollain, W. Burleson, F. G.

Moussy, A. Guiseppi-Elie, and G. De Micheli, Sensors, 2012, 12, 11013-11060.

9. F. Segura-Quijano, J. Sacristán-Riquelme, J. García-Cantón, M. T. Osés, and A. Baldi, Sensors (Basel)., 2010, 10, 4071-4082.

10. Z. Nie, F. Deiss, X. Liu, O. Akbulut, and G. Whitesides, Lab Chip, $2010,10,3163-3169$.

11. R. de la Rica and M. Stevens, Nat. Nanotechnol., 2012, 7, 821-824.

12. W. Li, X. Zhao, J. Zhang, and Y. Fu, Biosens. Bioelectron., 2014, 60, 252-258.

13. R. de la Rica and M. M. Stevens, Nat. Protoc., 2013, 8, 1759-1764.

14. B. Ngom, Y. Guo, X. Wang, and D. Bi, Anal. Bioanal. Chem., 2010, 397, 1113-1135.

15. C. Karakus and B. Salih, J. Immunol. Methods, 2013, 396, 8-14.

16. J. Hu, S. Wang, and L. Wang, Biosens. Bioelectron., 2014, 54, 585-597.

17. X. Mao, Y. Ma, A. Zhang, and L. Zhang, Anal. Chem., 2009, 81, $1660-1668$.

18

19

20

21.
G. Posthuma-Trumpie, J. Korf, and A. van Amerongen, Anal. Bioanal. Chem., 2009, 393, 569-582.

C. Parolo and A. Merkoçi, Chem. Soc. Rev., 2013, 42, 450-457.

C. Ge, L. Yu, Z. Fang, and L. Zeng, Anal. Chem., 2013, 85, $9343-$ 9349.

Z. Bao, S. Sun, J. Li, and X. Chen, Angew. Chem. Int. Ed. Engl., 2006, 45, 6723-6725.

L. Anfossi, C. Baggiani, and C. Giovannoli, Anal. Bioanal. Chem., 2013, 405, 467-480.

Y. Xia, J. Ye, K. Tan, J. Wang, and G. Yang, Anal. Chem., 2013, $\mathbf{8 5}, 6241-6247$.

D. Liu, Z. Wang, A. Jin, and X. Huang, Angew. Chem. Int. Ed., 2013, 52, 14065-14069.

J. Du, L. Jiang, Q. Shao, X. Liu, R. Marks, J. Ma, and X. Chen, Small, 2013, 9, 1467-1481.

S. Valetti, S. Mura, M. Noiray, S. Arpicco, F. Dosio, J. Vergnaud, D. Desmaële, B. Stella, and P. Couvreur, Bioconjug. Chem., 2014, 25, 1971-1983.

S. L. Capehart, A. M. ElSohly, A. C. Obermeyer, and M. B. Francis, Bioconjug. Chem., 2014, 25, 1888-1892.

J. Hui, A. Zaki, Z. Cheng, and V. Popik, Small, 2014, 10,33543363. 
29. M. Bloemen, T. Van Stappen, and P. Willot, PLoS One, 2014, 9, e109475.

30. V. Montes- García, J. Perez-Juste, I. Pastoriza-Santos, and L. M. Liz-Marzan, Chem. Eur. J., 2014, 20, 10874-10883.

31. X. Chen, S. G. Parker, G. Zou, W. Su, and Q. Zhang, ACS Nano, 2010, 4, 6387-6394

32. J. R. Waldeisen, T. Wang, B. M. Ross, and L. P. Lee, ACS Nano, $2011, \mathbf{5}, 5383-5389$.

33. F. Zheng, J. Wu, and G. Zhao, Anal. Methods, 2012, 4, 3932.

34. R. de la Rica and A. Velders, Small, 2011, 7, 66-69.

35. R. de la Rica and R. Fratila, Angew. Chem. Int. Ed. Engl., 2011, 50, 5704-5707.

36. A. Latorre and C. Posch, Chem. Commun., 2014, 50, 3018-3020.

37. H. Lee, S. Joo, S. Lee, and C. Lee, Biosens. Bioelectron., 2010, 26, $730-735$.

38. X. Yang and Z. Gao, Nanoscale, 2014, 6, 3055-3058.

39. A. Kanaras, Z. Wang, M. Brust, R. Cosstick, and A. Bates, Small, $2007,3,590-594$.

40. J. Liu, D. Wu, X. Yan, and Y. Guan, Talanta, 2013, 116, 563-568.

41. M. F. Cardinal, B. Rodrı, and L. M. Liz-marza, J. Phys. Chem. C, 2010, 114, 10417-10423.

42. L. Rodríguez-Lorenzo, R. de la Rica, R. Alvarez-Puebla, and L. M. Liz-Marzán, Nat. Mater., 2012, 11, 604-607.

43. A. Tsuboi, K. Nakamura, and N. Kobayashi, Adv. Mater., 2013, 25, $3197-3201$.

$44 . \quad$ L. Jing, K. Ding, S. Kershaw, I. M. Kempson, and A. L. Rogach, Adv. Mater., 2014, 26, 6367-6386.

45. K. Dohnalová, T. Gregorkiewicz, and K. Kusova, J. Phys. Condens. Matter, 2014, 26, 173201.

46. X. Ge, A. Asiri, D. Du, W. Wen, S. Wang, and Y. Lin, TrAC Trends Anal. Chem., 2014, 58, 31-39.

47. R. de la Rica, D. Aili, and M. Stevens, Adv. Drug Deliv. Rev., 2012, 64, 967-978.

48. X. Liu, M. Atwater, J. Wang, and Q. Huo, Colloids Surfaces $B$ Biointerfaces, 2007, 58, 3-7.

49. V. Chegel, O. Rachkov, A. Lopatynskyi, S. Ishihara, I. Yanchuk, Y. Nemoto, J. P. Hill, and K. Ariga, J. Phys. Chem. C, 2012, 116, $2683-2690$.

50. X. Nie, R. Huang, C. Dong, and L. Tang, Biosens. Bioelectron., 2014, 58, 314-319.
51. G. Huy, M. Zhang, P. Zuo, and B. Ye, Analyst, 2011, 136, 3289 3294.

52.

53. S. Gupta, H. Andresen, J. Ghadiali, and M. Stevens, Small, 2010, 6, $1509-1513$.

54. Y. Liu, M. Shipton, and J. Ryan, Anal. Chem, 2007, 79, 22212229.

55. S. M. Ansar, R. Haputhanthri, B. Edmonds, D. Liu, L. Yu, A. Sygula, and D. Zhang, J. Phys. Chem. C, 2011, 115, 653-660.

56. R. de la Rica and M. M. Stevens, Nat. Nanotechnol., 2012, 7, 821824

57. D. Liu, J. Yang, H. Wang, and Z. Wang, Anal. Chem., 2014, 86, $5800-5806$

58. D. Cecchin, R. de la Rica, R. E. S. Bain, M. W. Finnis, M. M. Stevens, and G. Battaglia, Nanoscale, 2014, 6, 9559-9562.

59. P. Wang, Y. Song, Y. Zhao, and A. Fan, Talanta, 2013, 103, 392 397.

60. Y. Yang, X. Zhong, Q. Zhang, and L. Blackstad, Small, 2014, 10, $1430-1437$.

61. R. Bera and C. Raj, J. Photochem. Photobiol. A Chem., 2013, 270, $1-6$.

62. X. Hou, S. Chen, J. Tang, Y. Xiong, and Y. Long, Anal. Chim Acta, 2014, 825, 57-62.

63. G. Wang, X. Zhu, H. Jiao, and Y. Dong, Anal. Chim. Acta, 2012 , 747, 92-98.

64. H. He, X. Xu, H. Wu, and Y. Jin, Adv. Mater., 2012, 24, 17361740.

65. L. Rodríguez-Lorenzo, J. M. Romo-Herrera, J. Perez-Juste, R. Alvarez-Puebla, and L. M. Liz-Marzan, J. Mater. Chem., 2011, 21, 11544-11549.

66. X.-H. Yang, J. Ling, J. Peng, Q.-E. Cao, Z.-T. Ding, and L.-C. Bian, Anal. Chim. Acta, 2013, 798, 74-81.

67. W. Sun, K. Jiao, and S. Zhang, Anal. Lett., 2000, 33, 2653-2675.

68. L.-N. Zhang, H.-H. Deng, F.-L. Lin, X.-W. Xu, S.-H. Weng, A.-L. Liu, X.-H. Lin, X.-H. Xia, and W. Chen, Anal. Chem., 2014, 86, $2711-2718$

69. J. Lan, W. Xu, Q. Wan, X. Zhang, and J. Lin, Anal. Chim. Acta, 2014, 825, 63-68.

70. H. Su, H. Zhao, F. Qiao, L. Chen, R. Duan, and S. Ai, Analyst, 2013, 138, 3026-3031.

71. W. Liu, H. Yang, Y. Ding, S. Ge, J. Yu, M. Yan, and X. Song, Analyst, 2014, 139, 251-258. 
72. A. Asati, S. Santra, and C. Kaittanis, Angew. Chem. Int. Ed. Engl, 2009, 48, 2308-2312.

73. K. Chaudhari, N. Chaudhari, and J. Yu, Catal. Sci. Technol., 2012, 2, 119 .

74. W. Luo, C. Zhu, S. Su, D. Li, Y. He, Q. Huang, and C. Fan, ACS Nano, 2010, 4, 7451-7458.

75. A. R. Clapp, I. L. Medintz, J. M. Mauro, B. R. Fisher, and M. G. Bawendi, J. Am. Chem. Soc., 2004, 126, 301-310.

76. Y. Cao, S. Shi, L. Wang, J. Yao, and T. Yao, Biosens. Bioelectron., 2014, 55, 174-179.

77. J. Yao, K. Zhang, H. Zhu, F. Ma, and M. Sun, Anal. Chem., 2013, 85, 6461-6468.

78. P. B. Tchounwou, W. K. Ayensu, N. Ninashvili, and D. Sutton, Environ. Toxicol., 2003, 18, 149-175.

79. J.-S. Lee, M. S. Han, and C. A. Mirkin, Angew. Chem. Int. Ed. Engl., 2007, 46, 4093-4096.

80. J. J. Storhoff, R. Elghanian, R. C. Mucic, C. A. Mirkin, and R. L. Letsinger, J. Am. Chem. Soc., 1998, 120, 1959-1964.

81. N. Yang, Y. Gao, Y. Zhang, Z. Shen, and A. Wu, Talanta, 2014, 122, 272-277.

82. F. Chai, C. Wang, T. Wang, L. Li, and Z. Su, ACS Appl. Mater. Interfaces, 2010, 2, 1466-1470.

83. J. Du, L. Jiang, Q. Shao, X. Liu, R. S. Marks, J. Ma, and X. Chen, Small, 2012, 9, 1467-1481.

84. X. H. Yang, J. Ling, J. Peng, Q. E. Cao, Z. T. Ding, and L. C. Bian, Anal. Chim. Acta, 2013, 798, 74-81.

85. X. Hou, S. Chen, J. Tang, Y. Xiong, and Y. Long, Anal. Chim. Acta, 2014, 825, 57-62.

86. I. B. Hirsch, D. Armstrong, R. M. Bergenstal, B. Buckingham, B. P. Childs, W. L. Clarke, A. Peters, and H. Wolpert, Diabetes Technol. Ther., 2008, 10, 232-244; quiz 245-6.

87. M. Mutlu, S. Mutlu, I. H. Boyaci, B. Alp, and E. Piskin, in Polymers in Sensors, American Chemical Society, 1998, vol. 690, pp. 5-57.

88. Y. Xianyu, J. Sun, Y. Li, Y. Tian, Z. Wang, and X. Jiang, Nanoscale, 2013, 5, 6303-6306.

89. A. Heller and B. Feldman, Chem. Rev., 2008, 108, 2482-2505.

90. J. Wang, Chem. Rev., 2008, 108, 814-825.

91. M.-S. Steiner, A. Duerkop, and O. S. Wolfbeis, Chem. Soc. Rev., 2011, 40, 4805-4839.

92. Q. Liu, H. Li, Q. Zhao, R. Zhu, Y. Yang, Q. Jia, B. Bian, and L. Zhuo, Mater. Sci. Eng. C. Mater. Biol. Appl., 2014, 41, 142-151.
93. J.-S. Lee, P. a Ulmann, M. S. Han, and C. A. Mirkin, Nano Lett., 2008, 8, 529-33.

94. Z. Mei, H. Chu, W. Chen, F. Xue, J. Liu, H. Xu, R. Zhang, and L. Zheng, Biosens. Bioelectron., 2013, 39, 26-30.

95. L. Zhu, G. Gamez, H. Chen, K. Chingin, and R. Zenobi, Chem. Commun., 2009, 559-61.

96

K. Ai, Y. Liu, and L. Lu, J. Am. Chem. Soc., 2009, 131, 94969497.

97. A. Lesniewski, M. Los, M. Jonsson-Niedziol̀åka, A. Krajewska, K Szot, J. M. Los, and J. Niedziolka-Jonsson, Bioconjug. Chem., 2014, 25, 644-648.

98. X. Wang, L. Wu, J. Ren, D. Miyoshi, N. Sugimoto, and X. Qu, Biosens. Bioelectron., 2011, 26, 4804-4809.

99. Z. Chen, Y. Tan, C. Zhang, L. Yin, H. Ma, N. Ye, H. Qiang, and Y. Lin, Biosens. Bioelectron., 2014, 56, 46-50.

100. L. Liang, Y. Long, H. Zhang, and Q. Wang, Biosens. Bioelectron., 2013, 50, 14-18.

101. C.-J. Kim, D.-I. Lee, C. Kim, K. Lee, C.-H. Lee, and I.-S. Ahn, Anal. Chem., 2014, 86, 3825-3833. 